\title{
Estabilidade robusta de sistemas lineares politópicos via programação linear utilizando o Teorema de Gershgorin *
}

\author{
Izabella O. Gomes, ${ }^{*}$ Ricardo C. L. F. Oliveira, ${ }^{*}$ \\ Pedro L. D. Peres* \\ * Faculdade de Engenharia Elétrica e de Computação, \\ Universidade Estadual de Campinas - UNICAMP \\ Av. Albert Einstein, 400, 13083-852, Campinas, SP, Brasil \\ (e-mail: $\{$ izabella, ricfow, peres\}@dt.fee.unicamp.br).
}

\begin{abstract}
This paper addresses the problem of robust stability analysis of continuous-time linear systems with polytopic uncertainties. As usual, the stability conditions are obtained from a quadratic Lyapunov function with a matrix that depends on the uncertain parameters, providing parameter-dependent linear matrix inequality (LMI) conditions. Unlike traditional methods from the literature, that use sufficient LMI conditions (relaxations) and semi-definite programming to solve the problem, the main novelty in this paper is the use of Gershgorin's Theorem and linear programming to build a certificate of robust stability, with an a posteriori test of the positivity of the Lyapunov matrix. The proposed strategy is compared with LMI conditions from the literature (solved by means of semi-definite programming) in a database of stable uncertain continuous-time systems, with several dimensions in terms of number of states and vertices. The results show that the method is competitive when compared with others in terms of performance, and more computationally efficient when the number of states of the systems grows.

Resumo: Este artigo aborda o problema de análise de estabilidade robusta de sistemas lineares contínuos no tempo com incertezas politópicas. Como usual, as condições para certificar a estabilidade são obtidas a partir de uma função de Lyapunov quadrática com matriz dependente dos parâmetros incertos, resultando em desigualdades matriciais lineares (em inglês, Linear Matrix Inequalities — LMIs) dependentes de parâmetros. Diferente dos métodos tradicionais da literatura, que usam condições suficientes na forma de LMIs (relaxações) e programação semidefinida para resolver o problema, propõe-se como principal novidade a utilização do Teorema de Gershgorin e de programação linear na construção de um certificado de estabilidade robusta, com o teste de positividade da matriz de Lyapunov realizado a posteriori. A estratégia proposta é comparada com condições na forma de LMIs da literatura (resolvidas por programação semidefinida) em uma base de dados com sistemas lineares incertos contínuos no tempo estáveis, com várias dimensões em termos de número de estados e vértices. Os resultados mostram que o método é competitivo com os demais em termos de desempenho, e mais eficiente computacionalmente quando o número de estados do sistema cresce.
\end{abstract}

Keywords: Robust stability, Linear Matrix Inequalities, Gershgorin's Theorem.

Palavras-chaves: Estabilidade robusta, Desigualdades Matriciais Lineares, Teorema de Gershgorin.

\section{INTRODUÇÃO}

Um tópico relevante em engenharia é o estudo de estabilidade de sistemas dinâmicos, com destaque para os métodos capazes de lidar com modelos que possuem parâmetros incertos. Em função do aumento da capacidade dos recursos computacionais e do desenvolvimento de técnicas numéricas cada vez mais sofisticadas para construir certificados de estabilidade, um grande avanço pode ser constatado

\footnotetext{
* O presente trabalho foi realizado com apoio da Coordenação de Aperfeiçoamento de Pessoal de Nível Superior - Brasil (CAPES) Código de Financiamento 001, CNPq e FAPESP (2017/18785-5).
}

nas publicações sobre o assunto nos últimos trinta anos. Destacam-se os métodos baseados em programação semidefinida, para tratar problemas de controle em geral, com particular protagonismo para o uso de desigualdades matriciais lineares (em inglês, Linear Matrix Inequalities LMIs) (Boyd et al. (1994); Oliveira and Peres (2010)).

A estabilidade de um sistema linear incerto e invariante no tempo pode ser verificada pelos autovalores da matriz dinâmica para todo valor que a incerteza possa assumir. No caso geral, a verificação do posicionamento de todos os autovalores exige o cômputo exaustivo dos autovalores da matriz dinâmica em um conjunto infinito, o que só pode ser 
feito de maneira aproximada. Neste contexto, as condições obtidas são apenas necessárias, mas não suficientes para certificar a estabilidade robusta. Por outro lado, quando o sistema incerto depende de um único parâmetro confinado a um intervalo limitado, existem técnicas baseadas em perturbação de autovalores (Chen et al. (2017)) ou mesmo LMIs (Tsiotras and Bliman (2006)) que podem dar respostas exatas sobre a estabilidade.

Uma maneira de se obter condições suficientes, e com maior eficiência computacional, para a análise de estabilidade de sistemas politópicos incertos consiste na aplicação da desigualdade de Lyapunov aos vértices do domínio incerto, utilizando a mesma matriz de Lyapunov. Este conceito é conhecido como estabilidade quadrática (Horisberger and Belanger (1976); Barmish (1985)), que tem como desvantagem a produção de condições conservadoras, principalmente quando a incerteza é invariante no tempo. Por este motivo, muito esforço tem sido empregado para se diminuir o conservadorismo das condições de estabilidade, por exemplo, utilizando funções de Lyapunov dependentes de parâmetros (de Oliveira et al. (1999); Trofino and de Souza (2001); Ramos and Peres (2001, 2002); Oliveira and Peres (2007)). Além disso, o conservadorismo também pode ser reduzido por meio da inserção de variáveis de folga (de Oliveira and Skelton (2001)) ou do subparticionamento do domínio incerto (Gonçalves et al. (2006)).

Atualmente, existem condições necessárias e suficientes que caracterizam a estabilidade robusta por meio de funções de Lyapunov com dependência polinomial nos parâmetros (Henrion et al. (2004); Chesi et al. (2005); Oliveira and Peres (2007); Oliveira et al. (2008)). Nesse caso, diversos métodos matemáticos e computacionais podem ser utilizados para a resolução do problema como, por exemplo, decomposição em soma de quadrados (Parrilo and Lall (2003); Papachristodoulou et al. (2013)), ou parametrizações baseadas em matrizes de Gram (Chesi et al. (2009)). Além disso, estratégias baseadas em testes de positividade para polinômios (usando, por exemplo, B-splines, que são uma base de funções polinomiais por partes (Hilhorst et al. (2016)), ou bases de Bernstein (Boudaud et al. (2008); Leth et al. (2016))) também podem ser utilizadas (Kamyar and Peet (2015)). Porém, o custo computacional requerido para a resolução dessas condições é muito alto, inviabilizando o tratamento de sistemas de grandes dimensões (muitos estados ou muitos parâmetros incertos). Este fato se deve, principalmente, ao número excessivo de variáveis de otimização e restrições (linhas de LMIs).

Este artigo propõe métodos para certificar a estabilidade robusta de sistemas lineares incertos invariantes no tempo por meio de funções de Lyapunov polinomiais homogêneas, porém sem utilizar programação semi-definida para resolver as LMIs. A estratégia consiste em utilizar condições LMIs bem conhecidas na literatura, mas substituindo a resolução tradicional com programação semi-definida ( $L M I$ solvers) por um conjunto de restrições lineares. Para este fim, é empregado o Teorema de Gershgorin, construindo-se restrições lineares sobre os elementos da LMI que garantem a negatividade (ou positividade). Embora as condições resultantes sejam apenas suficientes, a técnica permite o aumento da eficiência computacional em dois sentidos. O primeiro está relacionado ao fato de que os métodos de pontos interiores, largamente utilizados para resolver problemas de otimização linear, cônica e semi-definida, possuem uma eficiência maior ao lidar com programação linear (Ben-Tal and Nemirovski (2001)). Em segundo lugar o método permite que resolvedores de programação linear baseados no bem conhecido método simplex também possam ser empregados, aumentando ainda mais as dimensões possíveis de serem tratadas. A fim de diminuir o número de restrições do problema, outra proposta apresentada é a verificação da positividade da matriz de Lyapunov $a$ posteriori, resolvendo-se primeiro o problema sem a restrição de positividade da matriz de Lyapunov. Se uma solução é encontrada, verifica-se se a matriz de Lyapunov é definida positiva (com testes mais simples, que envolvem apenas cálculo de autovalores) e, em caso afirmativo, a estabilidade robusta é comprovada. Simulações computacionais, que comparam o método proposto com a resolução convencional (programação semi-definida por meio de solvers de LMIs) para uma base de sistemas lineares incertos invariantes no tempo garantidamente estáveis, mostram que o método proposto certifica a estabilidade robusta dos sistemas de forma competitiva, em termos da porcentagem de sistemas identificados como estáveis, porém com menor custo computacional. Os resultados fornecidos por este artigo são vantajosos ou equiparam-se aos demais, principalmente quando considerados sistemas com muitos estados e poucos parâmetros incertos.

Notação: O conjunto de matrizes reais com dimensão $n \times m$ é indicado por $\mathbb{R}^{n \times m}$, com elementos representados por $m_{i j}, i=1, \ldots, n ; j=1, \ldots, m$. A transposição da matriz $M$ é indicada por $M^{T} . M>0(M<0)$ simboliza que a matriz $M$ é definida positiva (negativa) e o símbolo * representa um bloco induzido por simetria em uma matriz.

\section{PRELIMINARES}

Sistemas lineares politópicos contínuos no tempo podem ser representados em espaço de estados como

$$
\dot{x}=A(\alpha) x
$$

sendo $x \in \mathbb{R}^{n}$ o vetor de estados e $A(\alpha) \in \mathbb{R}^{n \times n}$ a matriz dinâmica incerta com estrutura politópica, o que significa que $A(\alpha)$ pode ser escrita como uma combinação convexa de $N$ vértices conhecidos, isto é

$$
\begin{gathered}
A(\alpha)=\sum_{i=1}^{N} \alpha_{i} A_{i}, \quad \alpha \in \Lambda \\
\Lambda \triangleq\left\{\alpha \in \mathbb{R}^{N}: \sum_{i=1}^{N} \alpha_{i}=1, \alpha_{i} \geq 0, i=1, \ldots, N\right\} .
\end{gathered}
$$

A estabilidade robusta de (1) é assegurada se todos os autovalores de $A(\alpha)$ estiverem localizados no semiplano esquerdo aberto do plano complexo, ou seja, se todos os autovalores tiverem parte real negativa.

Uma condição de estabilidade robusta equivalente pode ser obtida a partir da existência da função de Lyapunov

$$
\begin{gathered}
\nu(x)=x^{T} P(\alpha) x, \\
\operatorname{com} P(\alpha)=P(\alpha)^{T}>0, P(\alpha) \in \mathbb{R}^{n \times n} \text { que verifique } \\
\dot{\nu}(x)=\dot{x}^{T} P(\alpha) x+x^{T} P(\alpha) \dot{x} \\
=x^{T}\left(A(\alpha)^{T} P(\alpha)+P(\alpha) A(\alpha)\right) x<0, \quad \alpha \in \Lambda,
\end{gathered}
$$

garantindo assim a estabilidade assintótica da origem para todo $\alpha \in \Lambda$. Em outras palavras, $A(\alpha)$ é Hurwitz estável 
para todo $\alpha \in \Lambda$ se, e somente se, existir $P(\alpha)=P(\alpha)^{T}>$ 0 tal que

$$
A(\alpha)^{T} P(\alpha)+P(\alpha) A(\alpha)<0, \quad \forall \alpha \in \Lambda .
$$

A condição (6) é uma LMI dependente de parâmetros, ou LMI robusta, e precisa ser verificada para todo $\alpha \in \Lambda$ (espaço de dimensão infinita). Porém, existem diversas abordagens (relaxações) para a construção de um conjunto finito de restrições na forma de LMIs, desigualdades ou equações algébricas que, no caso de haver solução factível, asseguram a factibilidade da LMI robusta para todo $\alpha \in$ $\Lambda$. Basicamente, impõe-se uma estrutura para $P(\alpha)$ e, a seguir, desenvolvem-se condições suficientes para que (6) seja válida em todo o domínio incerto.

A estabilidade quadrática consiste em atestar a estabilidade do sistema (1) por meio da construção de uma função de Lyapunov, como em (6), para o caso em que $P(\alpha)=P, \forall \alpha \in \Lambda$, conforme enuncia o próximo lema.

Lema 1. (Horisberger and Belanger (1976)) O sistema (1) é estável $\forall \alpha \in \Lambda$ se existir $P=P^{T}>0$ tal que

$$
A_{i}^{T} P+P A_{i}<0, \quad i=1, \ldots, N .
$$

No Lema 1, a estabilidade do sistema (1) é certificada para todo $\alpha \in \Lambda$, mesmo com as LMIs testadas apenas nos vértices do politopo. Este resultado é estendido para o caso em que $P(\alpha)$ tem estrutura com dependência afim, fornecendo o teste de estabilidade apresentado a seguir.

Lema 2. (Ramos and Peres (2002)) Se existirem matrizes $P_{i}=P_{i}^{T}>0, i=1, \ldots, N$ tais que

$$
\begin{gathered}
A_{i}^{T} P_{i}+P_{i} A_{i}<0, \quad i=1, \ldots, N \\
A_{i}^{T} P_{j}+P_{j} A_{i}+A_{j}^{T} P_{i}+P_{i} A_{j}<0, \\
\quad i=1, \ldots, N-1, \quad j=i+1, \ldots, N
\end{gathered}
$$

então uma matriz de Lyapunov dependente de parâmetros que certifica a estabilidade do sistema (1) para todo $\alpha \in \Lambda$ é dada por

$$
P(\alpha)=P(\alpha)^{T}, \quad P(\alpha)=\sum_{i=1}^{N} \alpha_{i} P_{i}, \quad \alpha \in \Lambda .
$$

Uma outra abordagem que pode ser utilizada para certificar a estabilidade de (1) com $P(\alpha)$ afim é baseada na introdução de variáveis de folga $\mathcal{X}=\left[\begin{array}{ll}X_{1} & X_{2}\end{array}\right]^{T}$, por meio do Lema de Finsler, em (6).

Lema 3. (de Oliveira and Skelton (2001)) Se existirem $P_{i}=P_{i}^{T}>0, i=1, \ldots, N$ e $X_{1}, X_{2}$ tais que

$\left[\begin{array}{cc}X_{1} A_{i}+A_{i}^{T} X_{1}^{T} & P_{i}-X_{1}+A_{i}^{T} X_{2}^{T} \\ P_{i}+X_{2} A_{i}-X_{i}^{T} & -X_{2}-X_{2}^{T}\end{array}\right]<0, \quad i=1, \ldots, N$ então $P(\alpha)$ dada por (8) é tal que (6) é verificada $\forall \alpha \in \Lambda$.

A aplicação do Lema de Finsler diretamente nas condições do Lema 2 produz mais uma condição.

Lema 4. (Leite and Peres (2003)) Se existirem $P_{i}=P_{i}^{T}>$ 0 e $X_{1 i}, X_{2 i}, i=1, \ldots, N$ tais que

$$
\begin{gathered}
{\left[\begin{array}{cc}
X_{1 i} A_{i}+A_{i}^{T} X_{1 i}^{T} & P_{i}-X_{1 i}+A_{i}^{T} X_{2 i}^{T} \\
P_{i}+X_{2 i} A_{i}-X_{1 i}^{T} & -X_{2 i}-X_{2 i}^{T}
\end{array}\right]<0, \quad i=1, \ldots, N} \\
{\left[\begin{array}{cc}
\Phi_{11} & \star \\
\Phi_{21} & \Phi_{22}
\end{array}\right]<0, \quad i=1, \ldots, N-1, \quad j=i+1, \ldots, N} \\
\Phi_{11}=A_{i}^{T} X_{1 j}^{T}+X_{1 j} A_{i}+A_{j}^{T} X_{1 i}^{T}+X_{1 i} A_{j},
\end{gathered}
$$

$$
\begin{aligned}
& \Phi_{21}=P_{i}+P_{j}-X_{1 i}^{T}-X_{1 j}^{T}+X_{2 j} A_{i}+X_{2 i} A_{j}, \\
& \Phi_{22}=-X_{2 i}-X_{2 i}^{T}-X_{2 j}-X_{2 j}^{T},
\end{aligned}
$$

então $P(\alpha)$ dada por (8) é uma função de Lyapunov dependente de parâmetros que certifica a estabilidade do sistema (1) para todo $\alpha \in \Lambda$.

A próxima definição é essencial para as contribuições deste artigo.

Definição 1. (Horn and Johnson (1985)) Seja $M=M^{T} \in$ $\mathbb{R}^{n \times n}$. A matriz $M$ é diagonalmente dominante se

$$
\left|m_{i i}\right| \geq R_{i}, \quad \forall i=1, \ldots, n
$$

e é estritamente diagonalmente dominante se

$$
\left|m_{i i}\right|>R_{i}, \quad \forall i=1, \ldots, n
$$

com

$$
R_{i}=\sum_{j=1 ; j \neq i}^{n}\left|m_{i j}\right|, \quad \forall i=1, \ldots, n
$$

definindo os raios dos discos $D_{i}$ de Gershgorin, centrados em $m_{i i}, i=1, \ldots, n$, isto é,

$$
D_{i}=\left\{z \in \mathbb{C}:\left|z-m_{i i}\right| \leq R_{i}\right\}, \quad i=1, \ldots, n .
$$

A Definição 1, derivada do Teorema de Gershgorin, afirma que todo autovalor de $M$ está dentro de algum disco de Gershgorin de $M$. Esse resultado é utilizado para produzir um teste suficiente para a positividade de uma matriz.

Lema 5. $M=M^{T} \in \mathbb{R}^{n \times n}$ é definida positiva se

$$
\begin{aligned}
& m_{i j}>0, \quad i=1, \ldots, n, \quad j=i+1, \ldots, n \\
& m_{i i}>\sum_{j=1 ; j \neq i}^{n} m_{i j}, \quad i=1, \ldots, n .
\end{aligned}
$$

Para os resultados do artigo, considere $X_{g}(\alpha)$ uma matriz polinomial homogênea de grau $g$ em $\alpha$. O caso $g=1$ equivale à matriz com dependência afim em $\alpha$ utilizada nos Lemas 2-4. Mais detalhes dessa notação podem ser encontrados em Oliveira and Peres (2007).

\section{RESULTADOS PRINCIPAIS}

Com base no Lema 5, são apresentadas na sequência condições suficientes na forma de um número finito de desigualdades lineares escalares para testar a positividade de uma matriz polinomial homogênea de grau genérico.

Lema 6 . Seja $M_{g}(\alpha)=M_{g}(\alpha)^{T}$ uma matriz polinomial homogênea de grau $g$ em $\alpha \in \Lambda$. $M_{g}(\alpha)$ é definida positiva se todas as matrizes coeficientes associadas a todos os monômios satisfizerem as condições do Lema 5, ou seja, forem definidas positivas.

A prova do Lema 6 é imediata, observando que todos os monômios são não negativos (todos os $\alpha_{i}$ são não negativos) e que as desigualdades escalares garantem que as matrizes coeficientes são definidas positivas. Note que no Lema 5 o módulo não é mais considerado na condição que define o disco de Gershgorin. Assim, a formulação do problema é simplificada, ao preço de algum conservadorismo.

Em função do teste de positividade estabelecido pelo Lema 6, formulou-se uma nova condição de estabilidade robusta, baseada puramente em restrições lineares, como mostra o próximo teorema. Para um resultado mais geral, a matriz de Lyapunov é considerada de grau genérico $g$. 
Note que, sempre que o sistema for estável, existe uma matriz polinomial homogênea de grau finito $g$ solução da desigualdade de Lyapunov (Oliveira and Peres (2007)).

Teorema 1. Se existirem $P_{g}(\alpha)=P_{g}(\alpha)^{T}$ e $Q_{g+1}(\alpha)=$ $Q_{g+1}(\alpha)^{T}$, tais que a positividade de $Q_{g+1}(\alpha)$ seja verificada utilizando as condições do Lema $6 \mathrm{e}$

$$
A(\alpha)^{T} P_{g}(\alpha)+P_{g}(\alpha) A(\alpha)+Q_{g+1}(\alpha)=0,
$$

então testam-se todos os coeficientes associados aos monômios de $P_{g}(\alpha)$. Se forem definidos positivos, o sistema (1) é robustamente estável.

A existência de uma solução para (9) garante que $A(\alpha)^{\prime} P_{g}(\alpha)+P_{g}(\alpha) A(\alpha)<0$, pois $Q_{g+1}(\alpha)$ é uma matriz definida positiva. Ademais, a factibilidade do teste $P_{g}(\alpha)>0$ realizado a posteriori certifica a estabilidade robusta do sistema. A variável $Q_{g+1}(\alpha)$ não é estritamente necessária, uma vez que as condições do Lema 6 podem ser aplicadas diretamente na desigualdade de Lyapunov, como propõe o próximo teorema.

Teorema 2. Se existir $P_{g}(\alpha)=P_{g}(\alpha)^{T}$ tal que todos os coeficientes matriciais do polinômio

$$
-\left(A(\alpha)^{T} P_{g}(\alpha)+P_{g}(\alpha) A(\alpha)\right)>0
$$

sejam definidos positivos utilizando as condições do Lema 5, então testam-se todos os coeficientes associados aos monômios de $P_{g}(\alpha)$. Se forem definidos positivos, o sistema (1) é robustamente estável.

Embora o Teorema 2 tenha menor complexidade que o Teorema 1 , devido à exclusão de $Q_{g+1}(\alpha)$, os experimentos numéricos mostram que a presença desta variável extra é crucial para propiciar melhores resultados, de certa forma compensando o conservadorismo associado às condições do Lema 5. Nos Teoremas 1 e 2, o teste da positividade de $P_{g}(\alpha)$ é realizado após uma solução ter sido obtida, e, portanto, todas as matrizes coeficientes são conhecidas, reduzindo a avaliação ao teste numérico dos autovalores. Como uma abordagem para melhorar o teste de positividade de $P_{g}(\alpha)$, pode-se utilizar relaxações de Pólya, isto é, testar os coeficientes de $\left(\alpha_{1}+\ldots+\alpha_{N}\right)^{d} P_{g}(\alpha)$, com $d$ (inteiro) crescente (Oliveira and Peres (2005)).

Os Teoremas 1 e 2, portanto, propõem abordagens para a certificação da estabilidade de sistemas contínuos lineares incertos utilizando apenas desigualdades e igualdades lineares escalares, com características de esparsidade. Essa estratégia possibilita o uso de resolvedores especializados em programação linear, como os que utilizam o método simplex (por exemplo, o CPLEX). Neste contexto, os resolvedores baseados em métodos de pontos interiores podem ser mais eficientes do que quando aplicados à programação semi-definida (Ben-Tal and Nemirovski (2001)).

\section{EXEMPLOS NUMÉRICOS}

As rotinas foram implementadas no Matlab (R2018a) utilizando o parser Yalmip (Löfberg (2004)) e os solvers SeDuMi (Sturm (1999)) e Mosek (MOSEK ApS (2015)), por meio da interface Robust LMI Parser (ROLMIP), versão 3.0(Agulhari et al. (2019)), em um computador Intel Core (TM) i7-7700 CPU @ 3.60GHz x 8 com 16GB RAM. Para os Teoremas 1 e 2, foi utilizado ROLMIP apenas para extrair os coeficientes das matrizes polinomiais homogê- neas e, então, construir as restrições escalares por meio do Lema 5.

Os resultados numéricos foram obtidos a partir de uma base de dados ${ }^{1}$ de sistemas incertos estáveis criados randomicamente nos quais o autovalor com maior parte real negativa $\lambda_{\max }$ (computado por meio de uma grade fina no espaço paramétrico) está posicionado em $-10^{-3}$ e -10 e $N$ vértices, com $N=2$ e 30 e $n=2,10$ e 20 estados.

As Tabelas 1 e 2 apresentam dados referentes à comparação entre os métodos propostos neste trabalho e condições da literatura (Lemas 1-4 da Seção 2, por programação semi-definida) para sistemas de 2 estados e 2 vértices, avaliando a eficiência dos solvers SeDuMi e Mosek.

Tabela 1. Número $V$ de variáveis escalares e $L$ de restrições (linhas de LMIs, no caso dos Lemas 1-4) para sistemas de 2 estados e 2 vértices utilizando os Lemas 1, 2, 3 e 4 e os

Teoremas 1 e 2 propostos com $g=2$.

\begin{tabular}{c|cccccc} 
& $\mathrm{L} 1$ & $\mathrm{~L} 2$ & $\mathrm{~L} 3$ & $\mathrm{~L} 4$ & $\mathrm{~T} 1$ & $\mathrm{~T} 2$ \\
\hline$V$ & 3 & 6 & 14 & 22 & 33 & 9 \\
$L$ & 12 & 20 & 40 & 56 & 36 & 12
\end{tabular}

Tabela 2. Porcentagem de sistemas estáveis $(e)$ e tempo de processamento computacional $(t)$ para sistemas de 2 estados e 2 vértices para os Lemas 1, 2, 3 e 4 e os Teoremas 1 e 2 propostos com $g=2$, com $\lambda_{\max }=\{-0.001,-10\}$ e os solvers SeDuMi e Mosek.

\begin{tabular}{c|l|ll|ll}
\multirow{2}{*}{} & solver & \multicolumn{2}{|c|}{ SeDuMi } & \multicolumn{2}{c}{ Mosek } \\
\cline { 2 - 6 } & $\lambda_{\max }$ & $e(\%)$ & $t(m s)$ & $e(\%)$ & $t(m s)$ \\
\hline \multirow{2}{*}{ L1 } & -0.001 & 93 & 20.2 & 93 & 1.7 \\
\hline \multirow{2}{*}{ L2 } & -10 & 96 & 12.8 & 96 & 1.4 \\
\hline \multirow{2}{*}{ L3 } & -0.001 & 99 & 21.3 & 99 & 2.8 \\
\hline \multirow{2}{*}{ L4 } & -0.001 & 99 & 20.9 & 94 & 2.2 \\
\hline & -10 & 100 & 17.9 & 100 & 1.7 \\
T1 & -0.001 & 99 & 23.4 & 97 & 2.4 \\
\hline \multirow{2}{*}{ T2 } & -10 & 100 & 19.9 & 100 & 2.3 \\
\hline & -0.001 & 86 & 10.9 & 95 & 1.9 \\
& & 99 & 3.4 & 99 & 1.2
\end{tabular}

Conforme mostra a Tabela 1, o Teorema 1 é o que utiliza mais variáveis para resolver o problema, seguido dos Lemas 4 e 3 e do Teorema 2, nessa ordem. Porém, os teoremas propostos (com $g=2$ ) usam uma quantidade menor ou igual de restrições que os lemas em comparação, exceto pelos Lemas 1 e 2 em relação ao Teorema 2. O aumento de $g$ pode melhorar a efetividade das condições propostas mas, por outro lado, implica em maior esforço computacional, com mais variáveis e restrições. Esse efeito é menos acentuado no Teorema 2, que não possui a variável auxiliar.

Embora a Tabela 1 mostre que, em alguns casos, os Teoremas 1 e 2 demandam mais restrições, vale lembrar que são desigualdades escalares, enquanto nas outras condições (Lemas 1-4) tratam-se de linhas de desigualdades matriciais. Isto justifica o fato de os custos computacionais dos

1 A base de dados utilizada pode ser acessada em: http://www.dt.fee.unicamp.br/ ricfow/programs/SBAI2019/ baseSistemasSBAI19.zip 
teoremas propostos serem, na maioria das vezes, menores que os dos lemas em comparação. Tais grandezas podem ser vistas na Tabela 2, em que, no melhor caso, o método proposto resolve o problema 5.85 vezes mais rápido que os demais utilizando o solver SeDuMi e 2 vezes mais rápido com o Mosek. Por ter sido o mais eficiente dos solvers nos testes, apenas o Mosek é utilizado a partir deste ponto.

Os Teoremas propostos certificam a estabilidade de uma quantidade menor de sistemas quando comparados com os Lemas 1-4, o que é explicado pela aplicação da condição de positividade do Lema 5 . Um número menor de sistemas estáveis é identificado para $\lambda_{\max }=-0.001$ (mais próximo de zero). Porém, para um autovalor máximo mais distante do eixo imaginário do plano complexo, os Teoremas propostos passam a ser competitivos.

Tabela 3. Porcentagem de sistemas estáveis $(e)$, tempo de processamento computacional $(t)$, número $V$ de variáveis escalares e $L$ de restrições (linhas de LMIs, no caso dos Lemas 1-4) para sistemas de 10 estados e 2 vértices para os Lemas 1, 2, 3 e 4 e para os Teoremas 1 e 2 propostos com $g=2$, com $\lambda_{\max }=\{-0.001,-10\}$.

\begin{tabular}{|c|c|c|c|c|c|}
\hline & $\lambda_{\max }$ & $e(\%)$ & $t(m s)$ & V & $L$ \\
\hline \multirow{2}{*}{ L1 } & -0.001 & 57 & 5.9 & \multirow{2}{*}{55} & \multirow{2}{*}{300} \\
\hline & -10 & 92 & 5.0 & & \\
\hline \multirow{2}{*}{ L2 } & -0.001 & 96 & 9.9 & \multirow{2}{*}{110} & \multirow{2}{*}{500} \\
\hline & -10 & 100 & 6.8 & & \\
\hline \multirow{2}{*}{ L3 } & -0.001 & 86 & 37.8 & \multirow{2}{*}{310} & \multirow{2}{*}{1000} \\
\hline & -10 & 100 & 33.6 & & \\
\hline \multirow{2}{*}{ L4 } & -0.001 & 94 & 86.0 & \multirow{2}{*}{510} & \multirow{2}{*}{1400} \\
\hline & -10 & 100 & 75.2 & & \\
\hline \multirow{2}{*}{$\mathrm{T} 1$} & -0.001 & 98 & 16.1 & \multirow{2}{*}{605} & \multirow{2}{*}{660} \\
\hline & -10 & 100 & 13.0 & & \\
\hline \multirow{2}{*}{$\mathrm{T} 2$} & -0.001 & 43 & 19.5 & \multirow{2}{*}{165} & \multirow{2}{*}{220} \\
\hline & -10 & 83 & 16.1 & & \\
\hline
\end{tabular}

A Tabela 3 apresenta os resultados obtidos para sistemas com 10 estados e 2 vértices. Nota-se que os Lemas 1 e 2 demandam tempos menores de resolução, porém o Teorema 1 com $g=2$ certifica mais sistemas estáveis que todos os demais métodos, sendo o menos conservador neste cenário. Em contrapartida, o Teorema 2 obteve a menor quantidade de sistemas estáveis. O fato do Teorema 1 apresentar melhores resultados que o Teorema 2 pode ser justificado pela presença de $Q_{g+1}(\alpha)$.

Tabela 4. Porcentagem de sistemas estáveis (e), tempo de processamento computacional $(t)$, número $V$ de variáveis escalares e $L$ de restrições (linhas de LMIs, no caso dos Lemas 14) para sistemas de 2 estados e 30 vértices para os Lemas 1, 2, 3 e 4 e para os Teoremas 1 e 2 propostos com $g=1$ e $\lambda_{\max }=-0.001$.

\begin{tabular}{l|llllll} 
& L1 & L2 & L3 & L4 & T1 & T2 \\
\hline$e(\%)$ & 75 & 91 & 85 & 91 & 84 & 65 \\
$t(m s)$ & 4.4 & 66.8 & 11.8 & 257.1 & 59.9 & 7.2 \\
$V$ & 3 & 90 & 98 & 330 & 2880 & 90 \\
$L$ & 124 & 495 & 600 & 7560 & 4185 & 1325
\end{tabular}

Na Tabela 4 (2 estados e 30 vértices), observa-se o conservadorismo dos teoremas propostos ao se aumentar o número de parâmetros incertos nos sistemas, visto que estes certificam uma quantidade maior de sistemas estáveis apenas quando comparados o Teorema 1 e o Lema 1. Por outro lado, o Teorema 2 mostra-se vantajoso, em termos de eficiência computacional, quando comparado com os demais métodos que também utilizam matrizes de Lyapunov afins (Lemas 2-4). O Teorema 1, diferente do Teorema 2, apresenta custo computacional maior que o Lema 3, podendo ser menos vantajoso também nesse quesito.

Tabela 5. Porcentagem de sistemas estáveis (e), tempo de processamento computacional $(t)$, número $V$ de variáveis escalares e $L$ de restrições (linhas de LMIs, no caso dos Lemas 14) para sistemas de 20 estados e 2 vértices para os Lemas 1, 2, 3 e 4 e para o Teorema 1 proposto, com $g=1$, para $\lambda_{\max }=-0.001$.

\begin{tabular}{l|lllll} 
& L1 & L2 & L3 & L4 & T1 \\
\hline$e(\%)$ & 70 & 100 & 80 & 90 & 100 \\
$t(m s)$ & 34.6 & 106.9 & 458.4 & 1428.5 & 101.6 \\
$V$ & 210 & 420 & 1220 & 2020 & 1680 \\
$L$ & 1200 & 2000 & 4000 & 5600 & 1890
\end{tabular}

A Tabela 5 apresenta os resultados para 20 estados e 2 vértices. Como é possível verificar, o Teorema 1 certifica a estabilidade de todos os sistemas, da mesma forma que o Lema 2, porém com o menor tempo computacional. Finalmente, é importante observar que o Teorema 1 é o mais vantajoso quanto ao custo computacional, se comparado com os métodos que também utilizam matrizes de Lyapunov afins, embora o número de variáveis sempre tenha sido maior (com exceção do caso da Tabela 5). Isso corrobora o argumento de que os métodos de pontos interiores possuem maior eficiência quando aplicados à programação linear.

\section{CONCLUSÃO}

Este artigo propôs uma nova abordagem para certificação de estabilidade de sistemas dinâmicos incertos contínuos no tempo. Ao contrário das propostas conhecidas, que resolvem este tipo de problema utilizando LMI solvers e programação semi-definida, a estratégia apresentada utiliza-se unicamente de restrições lineares escalares, com base no Teorema de Gershgorin. Como outra novidade, a positividade da matriz de Lyapunov não é imposta com restrições, mas sim verificada a posteriori. Foi utilizada uma base de sistemas garantidamente estáveis, a partir da qual obtiveram-se resultados computacionais, tanto dos teoremas propostos quanto de condições da literatura resolvidas por programação semi-definida. Foi mostrado que, para sistemas com um maior número de estados, a técnica proposta é competitiva com os demais métodos da literatura, tanto em relação à certificação de estabilidade dos sistemas, quanto à eficiência computacional. Entretanto, para sistemas com muitos parâmetros incertos, o conservadorismo é maior e também há desvantagens quanto ao custo computacional. A extensão da metodologia proposta para analisar a estabilidade de sistemas discretos, ou outras LMIs dependentes de parâmetros, é imediata.

\section{REFERÊNCIAS}

C. M. Agulhari, A. Felipe, R. C. L. F. Oliveira, and P. L. D. Peres. Algorithm 998: The Robust LMI Parser 
- A toolbox to construct LMI conditions for uncertain systems. ACM Trans. Math. Softw., 45(3):36:1-36:25, August 2019. http://rolmip.github.io.

B. R. Barmish. Necessary and sufficient conditions for quadratic stabilizability of an uncertain system. J. Optim. Theory and Appl., 46(4):399-408, August 1985.

A. Ben-Tal and A. Nemirovski. Lectures on modern convex optimization: analysis, algorithms, and engineering applications, volume 2 of MPS-SIAM Series on Optimization. SIAM \& MPS, Philadelphia, 2001.

F. Boudaud, F. Caruso, and M.-F. Roy. Certificates of positivity in the Bernstein basis. Discrete \& Comput. Geom., 39(4):639-655, June 2008.

S. Boyd, L. El Ghaoui, E. Feron, and V. Balakrishnan. Linear Matrix Inequalities in System and Control Theory. SIAM Studies in Applied Mathematics, Philadelphia, PA, 1994.

J. Chen, P. Fu, C.-F. Mendez-Barrios, S.-I. Niculescu, and H. Zhang. Stability analysis of polynomially dependent systems by eigenvalue perturbation. IEEE Trans. Autom. Control, 62(11):5915-5922, November 2017.

G. Chesi, A. Garulli, A. Tesi, and A. Vicino. Polynomially parameter-dependent Lyapunov functions for robust stability of polytopic systems: An LMI approach. IEEE Trans. Autom. Control, 50(3):365-370, March 2005.

G. Chesi, A. Garulli, A. Tesi, and A. Vicino. Homogeneous Polynomial Forms for Robustness Analysis of Uncertain Systems, volume 390 of Lecture Notes in Control and Information Sciences. Springer-Verlag, Berlin, Germany, 2009 .

M. C. de Oliveira and R. E. Skelton. Stability tests for constrained linear systems. In S. O. Reza Moheimani, editor, Perspectives in Robust Control, volume 268 of Lecture Notes in Control and Information Science, pages 241-257. Springer-Verlag, New York, NY, 2001.

M. C. de Oliveira, J. C. Geromel, and L. Hsu. LMI characterization of structural and robust stability: The discrete-time case. Lin. Alg. Appl., 296(1-3):27-38, June 1999

E. N. Gonçalves, R. M. Palhares, R. H. C. Takahashi, and R. C. Mesquita. New approach to robust $\mathcal{D}$-stability analysis of linear time-invariant systems with polytopebounded uncertainty. IEEE Trans. Autom. Control, 51 (10):1709-1714, October 2006.

D. Henrion, D. Arzelier, D. Peaucelle, and J. B. Lasserre. On parameter-dependent Lyapunov functions for robust stability of linear systems. In Proc. 43rd IEEE Conf. Decision Control, pages 887-892, Paradise Island, Bahamas, December 2004.

G. Hilhorst, E. Lambrechts, and G. Pipeleers. Control of linear parameter-varying systems using B-splines. In Proc. 55th IEEE Conf. Decision Control, pages 32463251, Las Vegas, NV, USA, December 2016.

H. P. Horisberger and P. R. Belanger. Regulators for linear, time invariant plants with uncertain parameters. IEEE Trans. Autom. Control, 21:705-708, 1976.

R. A. Horn and C. R. Johnson. Matrix Analysis. Cambridge University Press, Cambridge, MA, USA, 1985.

R. Kamyar and M. M. Peet. Polynomial optimization with applications to stability analysis and control Alternatives to sum of squares. Discrete Cont. Dyn. Syst. B, 20(8):2383-2417, October 2015.
V. J. S. Leite and P. L. D. Peres. An improved LMI condition for robust $\mathcal{D}$-stability of uncertain polytopic systems. IEEE Trans. Autom. Control, 48(3):500-504, March 2003.

T. Leth, C. Sloth, and R. Wisniewski. Lyapunov function synthesis - algorithm and software. In Proc. 2016 IEEE Int. Symp. on Comput. Aided Control Syst. Des., pages 641-647, Buenos Aires, Argentina, September 2016.

J. Löfberg. YALMIP: A toolbox for modeling and optimization in MATLAB. In Proc. 2004 IEEE Int. Symp. on Comput. Aided Control Syst. Des., pages 284-289, Taipei, Taiwan, September 2004. http://yalmip.github. io.

MOSEK ApS. The MOSEK optimization software, 2015. http: //www. mosek. com.

R. C. L. F. Oliveira and P. L. D. Peres. Stability of polytopes of matrices via affine parameter-dependent Lyapunov functions: Asymptotically exact LMI conditions. Lin. Alg. Appl., 405:209-228, August 2005.

R. C. L. F. Oliveira and P. L. D. Peres. Parameterdependent LMIs in robust analysis: Characterization of homogeneous polynomially parameter-dependent solutions via LMI relaxations. IEEE Trans. Autom. Control, 52(7):1334-1340, July 2007.

R. C. L. F. Oliveira and P. L. D. Peres. Análise e controle de sistemas lineares por meio de desigualdades matriciais lineares. In A. P. Feltrin, C. R. Minussi, M. C. M. Teixeira, and R. A. R. Lázaro, editors, Tutoriais do XVIII Congresso Brasileiro de Automática, pages 203229. Cultura Acadêmica, São Paulo, 2010. ISBN 97885-7983-061-7.

R. C. L. F. Oliveira, M. C. de Oliveira, and P. L. D. Peres. Convergent LMI relaxations for robust analysis of uncertain linear systems using lifted polynomial parameterdependent Lyapunov functions. Syst. Control Lett., 57 (8):680-689, August 2008.

A. Papachristodoulou, J. Anderson, G. Valmórbida, S. Prajna, P. Seiler, and P. A. Parrilo. SOSTOOLS: Sum of squares optimization toolbox for MATLAB. http://arxiv.org/abs/1310.4716, 2013. http: //www.cds.caltech. edu/sostools

P. A. Parrilo and S. Lall. Semidefinite programming relaxations and algebraic optimization in control. European J. Control, 9(2-3):307-321, 2003.

D. C. W. Ramos and P. L. D. Peres. A less conservative LMI condition for the robust stability of discrete-time uncertain systems. Syst. Control Lett., 43(5):371-378, August 2001.

D. C. W. Ramos and P. L. D. Peres. An LMI condition for the robust stability of uncertain continuous-time linear systems. IEEE Trans. Autom. Control, 47(4):675-678, April 2002.

J. F. Sturm. Using SeDuMi 1.02, a MATLAB toolbox for optimization over symmetric cones. Optim. Method Softw., 11(1-4):625-653, 1999. http: //sedumi. ie.lehigh.edu/.

A. Trofino and C. E. de Souza. Biquadratic stability of uncertain linear systems. IEEE Trans. Autom. Control, 46(8):1303-1307, August 2001.

P. Tsiotras and P.-A. Bliman. An exact stability analysis test for single-parameter polynomially-dependent linear systems. IEEE Trans. Autom. Control, 51(7):11611164, July 2006. 